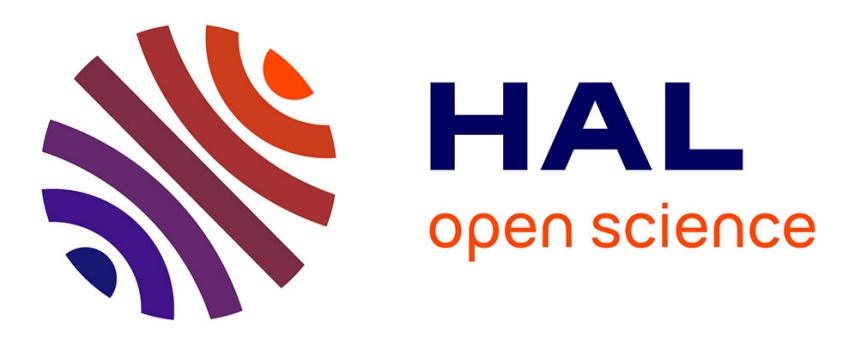

\title{
Serotypes and susceptibilities of paediatric clinical isolates of in Crete, Greece, before and after the heptavalent pneumococcal conjugate vaccine
}

\author{
S. Maraki, G. Samonis, E. Galanakis
}

\section{- To cite this version:}

S. Maraki, G. Samonis, E. Galanakis. Serotypes and susceptibilities of paediatric clinical isolates of in Crete, Greece, before and after the heptavalent pneumococcal conjugate vaccine. European Journal of Clinical Microbiology and Infectious Diseases, 2010, 29 (11), pp.1449-1451. 10.1007/s10096-0101005-0 . hal-00607639

\section{HAL Id: hal-00607639 \\ https://hal.science/hal-00607639}

Submitted on 10 Jul 2011

HAL is a multi-disciplinary open access archive for the deposit and dissemination of scientific research documents, whether they are published or not. The documents may come from teaching and research institutions in France or abroad, or from public or private research centers.
L'archive ouverte pluridisciplinaire HAL, est destinée au dépôt et à la diffusion de documents scientifiques de niveau recherche, publiés ou non, émanant des établissements d'enseignement et de recherche français ou étrangers, des laboratoires publics ou privés. 
Diseases

Editorial Manager(tm) for European Journal of Clinical Microbiology \& Infectious

Manuscript Draft

Manuscript Number: EJCMID-D-10-00179R1

Title: Serotypes and susceptibilities of paediatric clinical isolates of Streptococcus pneumoniae in Crete, Greece before and after the heptavalent pneumococcal conjugate vaccine

Article Type: Brief Report

Keywords: antimicrobial resistance; children; clinical isolates; Greece; Streptococcus pneumoniae; pneumococcal vaccine coverage

Corresponding Author: Dr. Emmanouil Galanakis,

Corresponding Author's Institution:

First Author: Sofia Maraki

Order of Authors: Sofia Maraki; George Samonis; Emmanouil Galanakis

Abstract: All Streptococcus pneumoniae strains isolated from paediatric clinical samples at Heraklion University General Hospital in the 10-year period 2000-2009 were tested for serotype and susceptibility to antimicrobials. Among a total of 258 strains, 159 were isolated in the 5-year period 2000-2004, before the heptavalent pneumococcal conjugate vaccine (PCV7) introduction and 99 in the post-PCV7 5-year period 2005-2009. Prevalence of PCV7-included serotypes decreased in the postPCV7 period $(\mathrm{p}=0.0002)$, but increase was observed for serotypes $7 F(p=0.002)$ and $19 A(p=0.004)$. Pan-susceptibility rates and susceptibility to cotrimoxazole increased in the post-PCV7 period $(\mathrm{p}=0.01$ and $p=0.008$, respectively), but serotype $19 \mathrm{~A}$ emerged as a contributor to multiresistance $(\mathrm{p}=0.007)$. PCV7 was followed by decreased S. pneumoniae resistance and prevalence of vaccine-related serotypes but increased prevalence of serotypes 7F and 19A. Continuing surveillance is required after the recent introduction of PCV10 and PCV13. 


\section{List of responses, Ms. No. EJCMID-D-10-00179}

Serotypes and susceptibilities of paediatric clinical isolates of Streptococcus pneumoniae in Crete, Greece before and after the heptavalent pneumococcal conjugate vaccine

\section{Reviewer \#1:}

Comment. This study examines the occurrence of individual pneumococcal serotypes and emergence of antimicrobial resistance, or not, before and after the introduction and widespread use of the pediatric seven-valent protein conjugated pneumococcal vaccine (PCV-7) on the Island of Crete. The group of invasive strains, blood, cerebrospinal fluid (CSF) and middle ear fluid, is only one-fourth of the total 258 strains reported and the respiratory tract strains represent more than one-half of the strains. It is difficult to judge whether a strain recovered from the respiratory tract is the pathogen or not. Thus, in published studies, PCV-7 vaccine efficacy has been estimated only using invasive strains. In this manuscript, analyzing invasive and non-invasive strains together distorts the effect of PCV-7 on invasive strains. Comment?

Response. We agree that up to date PCV-7 efficacy studies have mainly focused on invasive strains; however important studies investigating seroprevalence and resistance rates nasopharyngeal rate have appeared as well. In any case, in response to this comment we added (Results and Discussion, last paragraph, sentence 3): "The study included all clinical isolates rather than invasive strains or nasopharyngeal carriage samples alone, and this limitation did not allow for a reliable comparison of the PCV-7 efficacy results to previously reported findings."

Comment. The number of invasive strains recovered seems very low for ten years of surveillance. I estimated the pediatric population of Crete about 200,000 or about onethird of the total population. The low rates raises the question of whether all (or nearly all) patients admitted to hospital or treated in an emergency department for serious respiratory disease have blood cultures done. Comment?

Response. We do understand this comment; however the child population in Crete is actually 130,000. It is also true that the frequency of obtaining blood cultures does vary from country to country, but our study focused on the seroprevalence trends rather than the precise incidence per se. Nevertheless, following this comment we added data about the child population in Crete (Patients and Methods, paragraph 1, sentence 2): "The Hospital is the referral centre for the island of Crete with a coverage population of 631,000, of whom 130,000 are children aged less than 14 years."

Comment. Were physicians more likely to test for invasive pneumococcal strains in the five-year period after the introduction of vaccine as the numbers of strains were more in the second five-year period compared with the first five-year period even though the total number of strains recovered in the second five-year period was less than in the first fiveyear period?

Response. It is true that broad introduction of a vaccine increases awareness of the disease but we do not find that this awareness would only lead to increase of invasive strains and not of strains as a total. In order to stress this point and to avoid confusion we added (Results and Discussion, last paragraph, sentence 6): "Invasive disease and otitis media isolates appeared to be more common in the vaccine period, but it is questionable 
whether this increase should be attributed to the small sample size or to increased awareness of pneumococcal disease following the introduction of PCV7, rather than to a real increase in disease."

Comment. Abstract, page 2: In my copy of the manuscript, the notations between $\mathrm{p}$ and the value are missing. For example, line 9, p 0.0002, is $\mathrm{p}=0.0002$ meant here? Also, even though the authors note that "susceptibility rate increased for erythromycin," the $p$ value of 0.08 is not significant. Comment?

Response. Regarding $p$ values, we modified the text to " $p=0.0002$ " at the point suggested by Reiewer\#1 and, similarly, everywhere in the Abstract. Regarding the marginally significant difference in susceptibility to erythromycin, this information was deleted in the Abstract of the revised and abridged version of the manuscript.

Comment. Methods, page 4, paragraph 2: It is not clear from the text how soon after a patient was admitted that the blood culture was obtained. How many patients were treated in-hospital and how many as ambulatory patients?

Response. The retrospective nature of this study and the diverse origin of specimens from both the University Hospital itself and from other Health Care settings in Crete did not allow for reliable investigation of details such as precise sampling time or facility, or whether the child with a positive blood culture from the emergency department was not subsequently admitted.

Comment. Results, page 6, paragraph 2, line 6: What were the pus sites?

Response. The aforementioned limitation of the retrospective design of this study does not allow for reliable investigation of precise sampling site of pus.

Comment. Were the susceptibility patterns different for blood, CSF or middle ear fluid strains than ocular strains?

Response. Following this comment we re-calculated the susceptibilities patterns for ocular as compared to other isolates and these novel findings were added in Results Paragraph 3, sentence 7: "Ocular strains were more penicillin-sensitive than invasive and middle ear strains (72.1\% versus 52.2\%; p 0.01; OR 2.36, 95\% CI 1.04-5.47).”

Comment. Discussion: The Discussion could be shortened

Response. Discussion was quite dramatically shortened and incorporated in the "Results and Discussion" section of the revised manuscript, following both this comment and the editorial comment suggesting a manuscript in the format of a "Brief Report".

\section{Reviewer \#2:}

Comment. This is a single centre observational study of all pneumococcal strains from childhood samples collected at the University General Hospital, Heraklion, Crete, over a ten year period that included the introduction of 7-valent pneumococcal conjugate vaccine (PCV7). The main findings, of the decline in PCV7 serotype strains, and the increase in some non-PCV7 serotype strains, after the introduction of PCV7, have now been reported in many other publications. The unique aspect of this study is the reporting 
of data from Crete and there are few data on this topic from Greece or other Mediterranean areas. The other unique aspect is the apparent increase in isolation of pneumococcal isolates after the introduction of PCV7. However the authors provide no information to explain this apparent increase which may be an artefact.

Response. Following this point and a similar point by Reviewer\#1, we added (Results and Discussion, last paragraph, sentence 6): "Invasive disease and otitis media isolates appeared to be more common in the vaccine period, but it is questionable whether this increase should be attributed to the small sample size or to increased awareness of pneumococcal disease following the introduction of PCV7, rather than to a real increase in disease."

Comment. The first main limitation of this study is that the overall sample size from 10 years is small $(n=258)$ and the proportion of the isolates that came from invasive isolates is very small (28) so most of the data are from non-invasive isolates about which no clinical data is provided.

Response. We agree that this is a major limitation of our study; to accommodate this issue, we added (Results and Discussion, last paragraph, sentencew 2 and 3): "Limitations of this study include the small cohort of isolates and the retrospective design which did not allow for investigation of factors potentially affecting seroprevalence and susceptibility besides the introduction of PCV7. The study included all clinical isolates rather than invasive strains or nasopharyngeal carriage samples alone, and this limitation did not allow for a reliable comparison of the PCV-7 efficacy results to previously reported findings."

Comment. The second main limitation is that no demographic information is provided about the pediatric population on Crete, no clinical data is provided and there is no explanation of clinical and laboratory practices related to the collection of non-invasive specimens. So the reader cannot understand if the numbers of isolates obtained were influenced by any factors besides the introduction of PCV7.

Response. In response to this comment, we added information about the child population in Crete (Patients and Methods, paragraph 1, sentence 2): "The Hospital is the referral centre for the island of Crete with a coverage population of 631,000, of whom 130,000 are children aged less than 14 years." On the other hand it is true that the retrospective design does not allow for investigation of confounding factors potentially affecting pneumococcal seroprevalence besides the introduction of PCV7 and, as already mentioned, this was acknowledged in Results and Discussion, last paragraph, sentence 2): "Limitations of this study include the small cohort of isolates and the retrospective design which did not allow for investigation of factors potentially affecting seroprevalence and susceptibility besides the introduction of PCV7."

Some specific comments include the following:

Comment. Page 3/line 34. Do the authors have any data on PCV7 vaccination rates on Crete?

Response. No published data exist on PCV7 vaccination rates in Crete; however given the nationwide implementation of the vaccine we do believe that the rates from other areas of Greece apply to Crete. In response to this comment we modified the text 
(Introduction, first paragraph, sentence 3) to: "In Greece, PVC7 became available in 2004 and was introduced for all children younger than 5 years of age in January 2006. Vaccination compliance has been high nationwide with reported rates of up to $98 \%$ [5]."

Comment. Page 3/line 44. How were "children" defined? The results include and age range from 15 days to 14 years of age. What was the upper limit of age?

Response. Age definition was added (Patients and Methods, first paragraph, sentence 2): The Hospital is the referral centre for the island of Crete with a coverage population of 631,000 , of whom 130,000 are children aged less than 14 years.

Comment. Page 3/line 49ff. What were the clinical and laboratory practices related to collection of both invasive and non-invasive specimens over the course of the study and were there any changes? In addition, what was the trend in population of children on Crete during the study. Although this study does not report disease rates and is not population-based, the authors note the near doubling of invasive isolates in the period after PCV7 introduction compared with before (18/99 vs 10/159) as well as an increase in isolates from middle ear fluid (24/99 vs 15/159). Could these apparent increases be related to changes in clinical or laboratory practices, or population, as opposed to reflecting a real increase in disease? Are there clinical data about the children from whom the isolates were obtained?

Response. Due to the retrospective design of the study and due to the fact that specimens originated from more than one health care facilities we could not reliably investigate changes in collection of invasive and non-invasive specimens. In order to stress this uncertainty which was also noted by Reviewer\#1, we added (Results and Discussion, last paragraph 4, sentence 6): "Invasive disease and otitis media isolates appeared to be more common in the vaccine period, but it is questionable whether this increase should be attributed to the small sample size or to increased awareness of pneumococcal disease following the introduction of PCV7, rather than to a real increase in disease." Furthermore, as already stated, these limitations were appropriately stressed in Results and Discussion, last paragraph 4, sentence 2): Limitations of this study include the small cohort of isolates and the retrospective design which did not allow for investigation of factors potentially affecting seroprevalence and susceptibility besides the introduction of PCV7..

Comment. Page 7/line 31 and Table 2. It is incorrect to state that "rates of nonsusceptibility in the vaccine period were lower for the majority of antibiotics assayed..." There was a statistically significant $(\mathrm{P}<0.05)$ change for only one antibiotic (cotrimoxazole) and near-significant changes for 4 others.

Response. Following this comment we modified the text (Results and Discussion, paragraph 3, first sentence) to: "Non-susceptibility rates were significantly lower in the vaccine than in the pre-vaccine period for cotrimoxazole (20.2\% vs. $36.5 \%$; p 0.008 ; OR 0.44; 95\% CI 0.24-0.79) and near-significantly lower for erythromycin (p 0.081)." 
Serotypes and susceptibilities of paediatric clinical isolates of Streptococcus pneumoniae in Crete, Greece before and after the heptavalent pneumococcal conjugate vaccine

S. Maraki · G. Samonis · E. Galanakis

S. Maraki

Department of Clinical Bacteriology, Parasitology, Zoonoses and Geographic Medicine, University Hospital of Heraklion, Greece.

G. Samonis

Department of Internal Medicine, University of Crete, Greece

E. Galanakis $(\bowtie)$

Department of Paediatrics, University of Crete, 71003 Heraklion, Greece e-mail: emmgalan@med.uoc.gr 


\begin{abstract}
All Streptococcus pneumoniae strains isolated from paediatric clinical samples at Heraklion University General Hospital in the 10-year period 2000-2009 were tested for serotype and susceptibility to antimicrobials. Among a total of 258 strains, 159 were isolated in the 5-year period 2000-2004, before the heptavalent pneumococcal conjugate vaccine (PCV7) introduction and 99 in the post-PCV7 5-year period 20052009. Prevalence of PCV7-included serotypes decreased in the post-PCV7 period ( $\mathrm{p}=0.0002)$, but increase was observed for serotypes 7F $(\mathrm{p}=0.002)$ and 19A $(\mathrm{p}=0.004)$. Pan-susceptibility rates and susceptibility to cotrimoxazole increased in the post-PCV7 period ( $\mathrm{p}=0.01$ and $\mathrm{p}=0.008$, respectively), but serotype $19 \mathrm{~A}$ emerged as a contributor to multiresistance $(\mathrm{p}=0.007)$. PCV7 was followed by decreased $S$. pneumoniae resistance and prevalence of vaccine-related serotypes but increased prevalence of serotypes 7F and 19A. Continuing surveillance is required after the recent introduction of PCV10 and PCV13.
\end{abstract}




\section{Introduction}

The introduction of heptavalent pneumococcal conjugated vaccine (PCV7) has resulted in decrease of pneumococcal infections, but reports on increase of potentially invasive and resistant strains necessitate the surveillance of $S$. pneumoniae isolates [1]. Information on the impact of the implementation of PCV7 into the immunization programs comes mainly from the USA and Canada, and in a lesser degree from Europe [2-8]. In Greece, PVC7 became available in 2004 and was introduced for all children younger than 5 years of age in January 2006. Vaccination compliance has been high nationwide with reported rates of up to $98 \%$ [5]. Crete is the southernmost point of Europe and the second major island in the Eastern Mediterranean basin. In this study we investigated the circulating $S$. pmeumoniae serotypes and the trends in antimicrobial susceptibility of clinical isolates form children pre- and post the PCV7 introduction.

\section{Patients and methods}

This study included all paediatric S. pneumoniae specimens which were cultured, serotyped and tested for susceptibility to antimicrobials in the Laboratory of Microbiology at the University General Hospital, Heraklion, Greece for the 10-year period January 2000 through December 2009. The Hospital is the referral centre for the island of Crete with a coverage population of 631,000 , of whom 130,000 are children aged less than 14 years. Invasive pneumococcal disease isolates were recovered from blood or CSF, and otitis media isolates from middle ear fluid. One isolate per patient was identified and tested. Samples were immediately plated after collection on chocolate agar and Columbia agar plates (Becton Dickinson, Sparks, MD) supplemented with $5 \%$ sheep blood and incubated at $35^{\circ} \mathrm{C}$ in an atmosphere of $5 \% \mathrm{CO}_{2}$ in air for 24-72 hours. For blood cultures, the automated BacT/Alert system 
(BioMérieux, Durham, NC, USA) was used. S. pneumoniae isolates were identified on the basis of colonial and microscopic morphology, haemolytic activity on sheep blood agar medium, optochin susceptibility and bile solubility.

All isolates were serotyped by the Quellung reaction using pooled and selected factor antisera from Statens Seruminstitut (SSI, Copenhagen, Denmark). Nineteen antimicrobial agents including $7 \beta$-lactams (penicillin, cefuroxime, cefotaxime, ceftriaxone, cefepime, imipenem and meropenen), 3 macrolides (erythromycin, clarithromycin, and roxithromycin), 4 quinolones (ciprofloxacin, moxifloxacin, levofloxacin, and sparfloxacin), clindamycin, cotrimoxazole, tetracycline, chloramphenicol, and vancomycin were assayed by E-test (AB Biodisk, Solna, Sweden). Results were interpreted according to the 2008 Clinical and Laboratory Standards Institute criteria. S. pneumoniae ATCC 6305 (penicillin-sensitive) and $S$. pneumoniae ATCC 49619 (penicillin-resistant) were used as control strains. Data were compared using Fisher exact probability test. Odds ratios (OR) and 95\% confidence intervals (CI) were calculated. Statistical significance was set at two-tailed $\mathrm{p}<0.05$.

\section{Results and discussion}

A total of 258 clinical isolates were collected from 140 boys and 118 girls. Children age ranged 15 days to 14 (mean, 3.16; median, 2.0) years. Isolates represented blood (23), cerebrospinal fluid (5), middle ear fluid (39), nasopharyngeal swabs (132), ocular secretions (43), sputum (6), bronchoalveolar lavage (6), and pus (4). 159 (61.6\%) isolates were collected during the pre-vaccine 5-year period 2000-2004 and 99 (38.4\%) during the vaccine 5-year period 2005-2009. Blood and CSF isolates were more common in the vaccine period (18/99 vs. $10 / 159 ; 18.2 \%$ vs. $6.29 \%$; p 0.004 ; OR 3.31 , 95\% CI 1.46-7.51) as were middle ear fluid isolates (24/99 vs. $15 / 159 ; 24.2 \%$ vs. 
9.43\%; p 0.001; OR 3.31, 95\% CI 1.62-6.78); by contrast non-invasive isolates were more common during the pre-vaccine period (134/159 vs. $57 / 99 ; 84.3 \%$ vs. $57.6 \%$; p $<0.0001$, OR 3.95, 95\% CI 2.20-7.08).

In the vaccine period decrease was observed for serotypes included in the PCV7 (Table 1), but increase was observed for the PCV10-included 7F, the PCV13-included 19A, and for the total of serotypes not included in PCV7, PCV10, or PCV13 (25 vs. 21 isolates; $25.3 \%$ vs. $13.2 \%$; p 0.019; OR 2.22, 95\% CI 1.16-4.23). Coverage by PCV7 was $69.2 \%$ for the pre-vaccine period, but coverage for the vaccine period by the 7 valent, 10-valent, and 13-valent PCV were $45.5 \%, 56.6 \%$, and $75.5 \%$ of clinical isolates, respectively. Prevailing serotypes for invasive infection were 19F (3/10) in the pre-vaccine and 7F (7/18) in the vaccine period. The prevailing serotype for otitis media was $19 \mathrm{~F}$ both in the in the pre-vaccine (4/15), and in the vaccine period (10/24).

Non-susceptibility rates were significantly lower in the vaccine than in the prevaccine period for cotrimoxazole $(20.2 \%$ vs. $36.5 \%$; p 0.008 ; OR 0.44 ; $95 \%$ CI 0.24 0.79 ) and near-significantly lower for erythromycin (p 0.081). 124/258 isolates (48.1\%) were susceptible to all antibacterials tested, and this pan-susceptibility was more common in the vaccine than in the pre-vaccine period (58/99 vs. $66 / 159 ; 58.6 \%$ vs. 41.5\%; p 0.01, OR 1.99, 95\% CI 1.20-3.32). The overall high-level resistance rates were $17.1 \%$ for penicillin, $26.4 \%$ for cefuroxime and $0 \%$ for cefotaxime and ceftriaxone. Among 91 isolates resistant to erythromycin, phenotypes $\mathrm{M}, \mathrm{cMLS}_{\mathrm{B}}$, and $\mathrm{iMLS}_{\mathrm{B}}$ were detected in 62, 25, and 4 isolates respectively. Invasive and non-invasive isolates had similar susceptibility patterns. Ocular strains were more penicillin-sensitive than invasive and middle ear strains (72.1\% vs. 52.2\%; p 0.01; OR 2.36, 95\% CI 1.04-5.47). Ultrahigh penicillin resistance (MIC $4 \mu \mathrm{g} / \mathrm{mL}$ ) was observed in 11/258 isolates (4.28\%), of whom 9 were serotyped as 19F. Ciprofloxacin MIC of $\geq 3 \mathrm{mg} / \mathrm{L}$ was observed in 
13/258 isolates. Among common serotypes in the vaccine period, serotype 19A was often non-susceptible to penicillin, cefuroxime, and erythromycin $(84.6 \%, 69.2 \%$, and $76.9 \%$, respectively), but serotype $7 \mathrm{~F}$ was sensitive to all antimicrobials tested. Multiresistance (penicillin, erythromycin, cotrimoxazole and tetracycline) rates were similar in the pre-vaccine and the vaccine period (13.8\% and $15.2 \%$, respectively). The principal multiresistant serotype in the pre-vaccine period was $19 \mathrm{~F}(16 / 22,72.7 \%$ deceasing to $7 / 15,46.7 \%$ in the vaccine period). Serotype $19 \mathrm{~A}$ was a significant contributor to multiresistant strains in the vaccine as compared to the pre-vaccine period (5/15 vs. $0 / 22,33.3 \%$ vs. $0 \%$; p 0.007).

The introduction and the high uptake of PCV7 in the study area and the ability to centrally test clinical isolates provided the tools to investigate the effects of vaccine introduction in a well-defined population. Limitations of this study include the small cohort of isolates and the retrospective design which did not allow for investigation of factors potentially affecting seroprevalence and susceptibility besides the introduction of PCV7. The study included all clinical isolates rather than invasive strains or nasopharyngeal carriage samples alone, and this limitation did not allow for a reliable comparison of the PCV-7 efficacy results to previously reported findings. Serotypes of S. pneumoniae isolates were similar to these reported elsewhere in Greece [2-6]. In the pre-vaccine era, PCV7 coverage was $69.2 \%$ in the study area, but following decrease in vaccine-included serotypes, coverage by PCV7 in Crete dropped to $36.5 \%$ during the last 3 study years. Invasive disease and otitis media isolates appeared to be more common in the vaccine period, but it is questionable whether this increase should be attributed to the small sample size or to increased awareness of pneumococcal disease following the introduction of PCV7, rather than to a real increase in disease. Reports on invasive pneumococcal disease due to non-PCV7 serotypes have been controversial [7, 
8]. Non-susceptibility rates of $S$. pneumoniae isolates were similar to rates already reported elsewhere in Greece [3, 4]. No resistance was observed to fluoroquinolones, and third-generation cephalosporins were active throughout the study. The introduction of PCV10 and PCV13 will reasonably necessitate continued monitoring of $S$. pneumoniae seroprevalence, replacement of isolates by non-vaccine serotypes, and susceptibility patterns. 


\section{References}

1. Isaacman DJ, McIntosh ED, Reinert RR (2010) Burden of invasive pneumococcal disease and serotype distribution among Streptococcus pneumoniae isolates in young children in Europe: impact of the 7-valent pneumococcal conjugate vaccine and considerations for future conjugate vaccines. Int J Infect Dis 14:e197-209

2. Maraki S, Christidou A, Tselentis Y (2001) Antimicrobial resistance and serotype distribution of Streptococcus pneumoniae isolates from Crete, Greece. Int J Antimicrob Agents 17:465-469

3. Zissis NP, Syriopoulou V, Kafetzis D, Daikos GL, Tsilimingaki A, Galanakis E, Tsangaropoulou I (2004) Serotype distribution and antimicrobial susceptibility of Streptococcus pneumoniae causing invasive infections and acute otitis media in children. Eur J Pediatr 163:364-368

4. Paraskakis I, Kafetzis DA, Chrisakis A, Papavasilliou H, Kirikou H, Pangalis A, Tzouvelekis LS, Athanasiou T, Legakis NJ; National Surveillance Network for Pneumococcal Resistance (2006) Serotypes and antimicrobial susceptibilities of 1033 pneumococci isolated from children in Greece during 2001-2004. Clin Microbiol Infect $12: 490-493$

5. Grivea IN, Panagiotou M, Tsantouli AG, Syrogiannopoulos GA (2008) Impact of heptavalent pneumococcal conjugate vaccine on nasopharyngeal carriage of penicillinresistant Streptococcus pneumoniae among day-care center attendees in central Greece. Pediatr Infect Dis J 27:519-525

6. Grivea IN, Tsantouli AG, Chryssanthopoulou DC, Syrogiannopoulos GA (2010). Interaction of the heptavalent pneumococcal conjugate vaccine and the use of individual antibiotics among children on nasopharyngeal colonization with erythromycin-resistant Streptococcus pneumoniae. Eur J Clin Microbiol Infect Dis 29:97-105

7. Whitney CG, Pilishvili T, Farley MM, Schaffner W, Craig AS, Lynfield R, Nyquist AC, Gershman KA, Vazquez M, Bennett NM, Reingold A, Thomas A, Glode MP, Zell ER, Jorgensen JH, Beall B, Schuchat A (2006) Effectiveness of seven-valent pneumococcal conjugate vaccine against invasive pneumococcal disease: a matched case-control study. Lancet 368:1495-1502

8. Fenoll A, Granizo JJ, Aguilar L, Giménez MJ, Aragoneses-Fenoll L, Hanquet G, Casal J, Tarragó D (2009) Temporal trends of invasive Streptococcus pneumoniae serotypes and antimicrobial resistance patterns in Spain from 1979 to 2007. J Clin Microbiol 47:10121020 
Table 1 Serotype distribution of 258 clinical isolates of Streptococcus pneumoniae from children in Crete, 2000-2009

\begin{tabular}{|c|c|c|c|c|}
\hline Serotypes & $\begin{array}{l}\text { Total } \\
2000-2009 \\
\text { N (\%) }\end{array}$ & $\begin{array}{l}\text { Pre-PCV7 } \\
2000-2004 \\
\text { N }(\%)\end{array}$ & $\begin{array}{l}\text { Post-PCV7 } \\
2005-2009 \\
\text { N }(\%)\end{array}$ & p; OR $(95 \% \text { CI })^{\mathrm{a}}$ \\
\hline PCV7-included & $155(60.1)$ & $110(69.2)$ & $45(45.5)$ & $0.0002 ; 0.37(0.22-0.62)$ \\
\hline 4 & 0 & 0 & 0 & ns; na \\
\hline $6 \mathrm{~B}$ & $31(12.0$ & $20(12.6)$ & $11(11.1)$ & ns; $0.87(0.40-1.90)$ \\
\hline $9 \mathrm{~V}$ & $14(5.43)$ & $12(7.55)$ & $2(2.02)$ & $0.087 ; 0.25(0.06-1.53)$ \\
\hline 14 & $25(9.68)$ & $17(10.7)$ & $8(8.08)$ & ns; $0.73(0.30-1.77)$ \\
\hline $18 \mathrm{C}$ & $2(0.78)$ & $2(1.26)$ & 0 & ns; na \\
\hline $19 \mathrm{~F}$ & $64(24.8)$ & $43(27.0)$ & $21(21.2)$ & ns; $0.73(0.40-1.32)$ \\
\hline $23 \mathrm{~F}$ & $19(7.36)$ & $16(10.1)$ & $3(3.03)$ & $0.048 ; 0.28(0.08-0.98)$ \\
\hline PVC7-not included & $86(33.3)$ & $39(24.5)$ & $47(47.5)$ & $0.0002 ; 2.78(1.62-4.75)$ \\
\hline $1^{\mathrm{b}}$ & 0 & 0 & 0 & ns; na \\
\hline $3^{\mathrm{c}}$ & $27(10.5)$ & $21(13.2)$ & $6(6.06)$ & $0.09 ; 0.42(0.17-1.09)$ \\
\hline $5^{\mathrm{b}}$ & 0 & 0 & 0 & ns; na \\
\hline $6 A^{c}$ & $3(1.16)$ & $1(0.63)$ & $2(2.02)$ & $\mathrm{ns} ; 3.26(0.29-36.4)$ \\
\hline $7 \mathrm{~F}^{\mathrm{b}}$ & $14(5.43)$ & $3(1.89)$ & $11(11.1)$ & $0.002 ; 6.50(1.77-23.9)$ \\
\hline $19 A^{c}$ & $13(5.04)$ & $3(1.89)$ & $10(10.1)$ & $0.004 ; 5.84(1.57-21.8)$ \\
\hline Other & $29(11.2)$ & $11(6.92)$ & $18(18.2)$ & $0.008 ; 2.99(1.35-6.64)$ \\
\hline Non-typeable & $17(6.59)$ & $10(6.29)$ & $7(7.07)$ & ns; $1.13(0.42-3.08)$ \\
\hline Total isolates & $258(100)$ & $159(100)$ & $99(100)$ & \\
\hline
\end{tabular}

${ }^{a}$ isolates in the period 2005-2009 as compared to isolates during 2000-2004; ${ }^{b}$ serotype included in the PCV10; ${ }^{\mathrm{c}}$ serotype included in the PCV13; na, not appropriate; ns, not significant 